\title{
LA REGULACIÓN DEL SISTEMA FINANCIERO: UN MODELO DE CRECIMIENTO
}

Salvador Rivas Aceves*

Departamento de Economía, Universidad Autónoma Metropolitana-Azcapotzalco

(Recibido 26 de abril 2013, aceptado 28 de junio 2013)

\section{Resumen}

En este trabajo se identifican los efectos que tiene el sistema financiero sobre la tasa de crecimiento de una economía. Para ello en un modelo de crecimiento endógeno estocástico se introducen dos tipos de sistemas financieros, uno eficiente y otro ineficiente, se analizan los efectos de ambos sobre el crecimiento y se encuentra que la ineficiencia financiera genera impactos negativos en el crecimiento. La regulación financiera vía un impuesto al rendimiento del capital corrige lo anterior y se encuentran las condiciones necesarias para crecer bajo este escenario. Se determina un recorrido óptimo para el impuesto y mediante simulación Monte Carlo se muestra que la regulación en efecto corrige las distorsiones generadas por ineficiencia financiera.

\begin{abstract}
The effects of a financial market on growth are studied. By using a model of stochastic endogenous growth with two types of financial markets (efficient and inefficient) the macroeconomic equilibrium is characterized and shows that negative impacts on growth appear when financial market is inefficient. Therefore regulation on financial market can be applied, by a capital revenue tax, in order to restore macroeconomic equilibrium. An optimal interval for the tax is defined and through Monte Carlo simulation the impact on growth due regulation are shown.

Clasificación JEL: G10, G18, G14, O40, E44.

Palabras clave: Mercado Financiero, Regulación, Eficiencia del Mercado de Capitales, Crecimiento Económico, Equilibrio Macroeconómico.
\end{abstract}

* Privada de Santa Mónica, Mz 5, Lt 14, A-703. Fraccionamiento Colinas de San José. Tlanepantla de Baz. Estado de México. C. P. 54190. Correo electrónico: rivas.salvador@gmail.com 


\section{Introducción}

Con la crisis financiera y económica, de tipo mundial, que se originó a finales del 2008 en Estados Unidos de Norte América quedó claro que existe una relación entre el sistema financiero y la economía real, esclareciendo así el panorama teórico al respecto. Todo comenzó con los desarrollos teóricos realizados por Bagehot (1873), Gurley y Shaw (1955) y McKinnon (1973) que mostraron que la relación entre las finanzas y el crecimiento existe. Más todavía, argumentan que un sistema financiero bien desarrollado puede generar impactos positivos sobre la economía real.

La palabra clave de su argumentación es "bien desarrollados", puede que no sea evidente pero un sistema financiero sólido presenta pocas o nulas ineficiencias lo que se traduce en una reasignación de recursos eficientes, como se mostrará en este ensayo, lo cual representa la función básica de un sistema financiero. Sin embargo, la existencia de ineficiencias al interior de dicho mercado puede ocasionar la aparición de restricciones al crédito, lo que ocasiona modificaciones en la composición del bienestar económico generando así inequidad. Lo anterior puede ocurrir o no, todo dependerá de las características propias del sistema financiero en concordancia con su entorno económico.

De aquí surgen dos vertientes, puede ser que el sector financiero promueva la acumulación del capital físico y del capital humano bajo la presencia de restricciones al crédito, y así estimular el crecimiento, como lo muestran los estudios realizados por Banerjee y Newman (1993), Galor y Zeira (1993), Aghion y Bolton (1997) y Piketty (1997), o en contra parte, las restricciones al crédito y cualquier otra ineficiencia financiera en realidad impiden el proceso de acumulación de capital y, por lo tanto, tiene un impacto negativo sobre el crecimiento económico como muestran Benabou (1993, 1996a, 1996b), Durlauf (1996a, 1996b), Fernández y Rogerson (1994, 1996), Kremer y Maskin (1994) y Rivas-Aceves y Martínez (2011).

Se verá en el presente documento que, la relación existente entre el sistema financiero y el sector real de la economía es favorable, positiva o directa, si las restricciones al crédito e ineficiencias del sector financiero no están presentes. Lo que concuerda con los resultados encontrados por la segunda vertiente descrita anteriormente. En consecuencia, el tipo de vínculo existente es de índole negativa cuando el sistema financiero no reasigna eficientemente los recursos en su poder, impactando de manera negativa sobre el crecimiento de la economía real.

Los efectos distorsionadores que el sistema financiero causa sobre la economía real pueden ser compensados por la regulación gubernamental al interior del mercado de capitales como lo sostienen Barth, Caprio y Levine (2001a, 2001b, 2004 y 2005), La Porta, López-de-Silanes y Shleifer (2005). En consecuencia, las regulaciones y las prácticas de supervisión impulsan el desarrollo del mercado de capitales. El análisis aquí realizado amplía esta última idea al mostrar que no sólo genera una mayor eficiencia en el sistema financiero, sino que también estimula el crecimiento.

Por último, hay estudios que verifican que la política monetaria y la política fiscal pueden promover la eficiencia de los servicios financieros, véase Bencivenga 
y Smith (1992), Huybens y Smith (1999), y Roubini y Sala-i-Martin (1992, 1995). Este fenómeno en particular no es de interés para el presente análisis.

Basándose en la idea de que un mercado con crédito puede aumentar el crecimiento económico debido a que permite la reasignación del capital productivo al interior del sector privado, Kunieda (2008), es de interés mostrar cómo la economía en equilibrio crece a una tasa de crecimiento balanceado y de pleno empleo, lo que realiza en el apartado 1. En el apartado 2 se muestra que dicho equilibrio puede o no ser modificado en función de las características del sistema financiero, es decir si presenta ineficiencias o no, lo que ocasiona impactos negativos y positivos, respectivamente, sobre el crecimiento.

Si el sistema financiero es ineficiente, los efectos negativos causados pueden ser corregidos mediante la intervención del gobierno a través de una regulación financiera, al imponer un impuesto al costo excesivo del capital, lo que se presenta en el apartado 3. Se realiza una simulación de tipo Monte Carlo respecto del modelo propuesto en el aparatado 4. Los resultados se describen en el apartado 5 y muestran que: 1) existe un vínculo entre sector financiero y sector real, 2) la dirección y la magnitud de dicha relación dependen de las características del sistema financiero, 3) si el sistema financiero impacta negativamente sobre el crecimiento, la regulación financiera revierte dicho fenómeno, y 4) existe un comportamiento óptimo del impuesto aplicado al costo del capital que permite regresar a una senda de crecimiento sostenido, balanceado y de pleno empleo. Finalmente, presentar un anexo matemático en el apartado 6 y enlistar las referencias en el aparatado 7 .

\section{La Economía}

Suponga que la economía está compuesta por agentes económicos idénticos, es decir, con preferencias y dotaciones iniciales iguales para todos y con un horizonte de vida infinito para cada poblador, lo cual implica que cada agente se preocupa por las decisiones que toma a lo largo de su vida, así como por las que tomará toda su descendencia. Al interior de la economía se produce un único bien de consumo que es perecedero que no se puede comerciar con el resto del mundo, porque inicialmente se considera cerrada a la economía. Bajo estas condiciones la economía se comporta de la siguiente manera.

\subsection{Hogares}

Los hogares están compuestos por consumidores que buscan maximizar el valor esperado de su utilidad debido al consumo del bien perecedero, el cual está medido por:

$$
E \int_{0}^{\infty} u\left(c_{t}\right) e^{-\rho t} d t
$$

en donde la función de utilidad $u\left(c_{t}\right)$ cumple con que $u^{\prime}\left(c_{t}\right)>0 \mathrm{y} u^{\prime \prime}\left(c_{t}\right)<0$, en otras palabras, presenta rendimientos marginales decrecientes.

Bajo esta definición, el consumo per cápita está representado por $c_{t} \mathrm{y}$ $\rho$ es la tasa subjetiva de descuento que mide las preferencias por el consumo presente del individuo representativo. Suponga una utilidad logarítmica del tipo $u\left(c_{t}\right)=\ln c_{t} \mathrm{y}$, además, que el consumidor tiene acceso a información para la 
toma de decisiones, la cual está disponible en cada momento $t$. En consecuencia, la función de utilidad de tipo von Neumann-Morgenstern separable en $t=0$ que mide dicho comportamiento es:

$$
\mathcal{V}_{0}=E\left\{\int_{0}^{\infty} \ln c_{t} e^{-\rho t} d t \mid \mathcal{F}_{0}\right\}
$$

con $\mathcal{F}_{0}$ como el conjunto de información inicial o las condiciones iniciales de las variables económicas, el cual será definido más adelante.

\subsection{Aparato productivo}

Los productores utilizan todo el capital disponible, $k_{t}$, para producir el bien de consumo, y se considera disponible en el sentido de que es aquel que no ha sido consumido, lo que implica que el bien de consumo puede utilizarse como bien de capital. Asimismo, la producción per cápita, que está cuantificada por $y_{t}$, se lleva a cabo a través de una tecnología dada para todos los productores, de tal forma que las condiciones de producción son:

$$
d y_{t}=\alpha\left(k_{t} d t+k_{t} \sigma_{y} d W_{t}+k_{t} \nu_{y} d Z_{t}\right),
$$

en donde $\alpha$ mide la media esperada de la productividad marginal del capital, $\sigma_{y}$ la dispersión en la productividad esperada y $\nu_{y}$ un salto en la productividad. Por su parte, $d W_{t}$ es un proceso de Wiener definido en un espacio fijo de probabilidad cuya filtración aumentada cumple que $\left(\Omega, F,\left(F_{t}\right)_{t \geq 0}, \mathbb{P}\right)$, con incrementos temporales independientes, media cero y varianza igual al incremento temporal, mientras que $d Z_{t}$ es un proceso de Poisson que caracteriza la dinámica del salto en la productividad con una intensidad $\theta_{y}$ de manera que:

$\left\{\begin{array}{l}\operatorname{Pr}(\text { un salto durante } d t)=\operatorname{Pr}\left(d Z_{t}=1\right)=\theta_{y} d t \\ \operatorname{Pr}(\text { ningun salto durante } d t)=\operatorname{Pr}\left(d Z_{t}=0\right)=1-\theta_{y} d t+o(d t) \\ \operatorname{Pr}(\text { ms de un salto durante } d t)=\operatorname{Pr}\left(d Z_{t}>1\right)=o(d t)\end{array}\right.$

Se supone adicionalmente que $d W_{t}$ y $d Z_{t}$ no están correlacionados, que el número inicial de saltos en la productividad es $Z_{0}=0$, que existe un nivel de capital inicial positivo, $k_{0}$, y además se considera que $o(d t)$ mide efectos de las variables en cuestión que persistan más allá de $t+d t$, los cuales son insignificantes y con $o(d t) / t \rightarrow 0$ cuando $t \rightarrow 0$.

\subsection{Comportamiento agregado}

Suponga que los agentes económicos toman de manera simultánea decisiones de producción y de consumo, en otras palabras, que los hogares son los dueños de los medios de producción. En consecuencia, la producción total en la economía se destina o a la inversión o al consumo, es decir:

$$
d y_{t}=d k_{t}+c_{t} d t
$$


Al sustituir la ecuación (3) en (7) se encuentra la identidad contable que determina la dinámica de la acumulación del capital para cada agente económico representativo, por lo tanto:

$$
\frac{d k_{t}}{k_{t}}=\alpha\left(d t+\sigma_{y} d W_{t}+\nu_{y} d Z_{t}\right)-\frac{c_{t}}{k_{t}} d t
$$

Equivalentemente,

$$
d k_{t}=k_{t}\left(\alpha-\frac{c_{t}}{k_{t}}\right) d t+k_{t} \alpha \sigma_{y} d W_{t}+k_{t} \alpha \nu_{y} d Z_{t} .
$$

La toma decisiones de los agentes involucra el conocimiento, de manera simultánea, de la restricción establecida en (8') y de la información disponible en $t=0$ determinada por $\mathcal{F}_{0}=\left\{k_{0}, Z_{0}\right\}$

\subsection{Equilibrio macroeconómico}

Bajo las condiciones establecidas, el agente representativo toma sus decisiones en función de los resultados arrojados por la solución del problema de control óptimo estocástico determinado por las ecuaciones (2) y (8'). Dicha solución, (véase el apéndice matemático), está dada por la condición Hamilton-JacobiBellman y sus condiciones de optimalidad siguientes:

$$
\begin{gathered}
0=\ln c_{t}-\rho V\left(k_{t}\right)+k_{t} V^{\prime}\left(k_{t}\right)\left(\alpha-\frac{c_{t}}{k_{t}}\right)+\frac{1}{2} V^{\prime \prime}\left(k_{t}\right) \alpha^{2} k_{t}{ }^{2} \sigma_{y}^{2} \\
+\left[V\left(k_{t}\left(1+\alpha \nu_{y}\right)\right)-V\left(k_{t}\right)\right] d Z t, \\
\frac{1}{c_{t}}-V^{\prime}\left(k_{t}\right)=0, \\
V\left(k_{t}\right)=\beta_{0}+\beta_{1} \ln k_{t}, \\
V^{\prime}\left(k_{t}\right)=\beta_{1} / k_{t}, \\
V^{\prime}\left(k_{t}\right)=-\beta_{1} / k_{t}{ }^{2}, \\
c_{t}=\frac{k_{t}}{\beta_{1}} .
\end{gathered}
$$

De las condiciones de optimalidad $\beta_{0}$ y $\beta_{1}$ son constantes a determinar. La ecuación (14) muestra que se consume siempre una proporción fija del capital, y dado que sólo existe un único bien que se destina ya sea al consumo o la inversión, entonces la proporción fija que no se consume representa la inversión. Para determinar $\beta_{1}$ es necesario sustituir la función $V\left(k_{t}\right)$ y sus correspondientes derivadas de primer y segundo orden (condiciones 11-13) en la ecuación (9), además suponer que $\left(1-\rho \beta_{1}\right)=0$, y por último considerar la condiciones establecidas en la ecuaciones (4)-(6). De esta forma se obtiene:

$$
0=\ln \rho-\beta_{0} \rho+\frac{\alpha}{\rho}-\frac{\alpha^{2} \sigma_{y}^{2}}{2 \rho}-1+\frac{\alpha \theta_{y}}{\rho} \ln \left(1+\nu_{y}\right)
$$


En consecuencia, al considerar las condiciones anteriores se tiene que el equilibrio macroeconómico es:

$$
\begin{gathered}
\beta_{1}=\frac{1}{\rho} \\
\beta_{0}=\frac{1}{\rho}\left[\ln \rho+\frac{\alpha}{\rho}+\frac{\alpha \theta_{y}}{\rho} \ln \left(1+\nu_{y}\right)-\frac{\alpha^{2} \sigma_{y}^{2}}{2 \rho}-1\right] \\
k_{t}=k_{0} e^{\left(\alpha-\rho-\frac{\alpha^{2} \sigma_{y}^{2}}{2}\right) t+\alpha \sigma_{y} W_{t}+\ln \left(1+\alpha \nu_{y}\right) Z_{t}}, \\
c_{t}=\rho k_{0} e^{\left(\alpha-\rho-\frac{\alpha^{2} \sigma_{y}^{2}}{2}\right) t+\alpha \sigma_{y} W_{t}+\ln \left(1+\alpha \nu_{y}\right) Z_{t}}, \\
\psi=\left(\alpha-\rho-\frac{\alpha^{2} \sigma_{y}^{2}}{2}\right) t+\alpha \sigma_{y} \xi+\ln \left(1+\alpha \nu_{y}\right) Z_{t}
\end{gathered}
$$

en donde $\xi=W_{t}$ y además $\xi \sim \mathcal{N}(0,1)$. Se puede apreciar que las proporciones $\beta_{1}$ y $\beta_{0}$ son invariantes en el tiempo, que el nivel de capital depende sólo del nivel de capital inicial y que el consumo per cápita está directamente determinado por las preferencias de los consumidores y por la trayectoria de equilibrio del capital.

\subsection{Tasa de crecimiento económico}

El crecimiento de esta economía depende de cómo crezca la acumulación del capital, condición determinada por la trayectoria de equilibrio establecida en (18). Asimismo, la ecuación (20) muestra de manera detallada el dinamismo en el crecimiento, el cual presenta dos componentes: el determinista y el estocástico. Al considerar la ecuación (8) y la ecuación (20) se tiene que el componente determinista es:

$$
\Psi=E\left[\frac{d k_{t}}{k_{t}} \cdot \frac{1}{d t}\right]=\alpha+\alpha \nu_{y} \theta_{y}-\rho .
$$

Para que la economía crezca es necesario que siempre se cumpla que $\alpha+$ $\alpha \nu_{y} \theta_{y}>\rho$, en caso contrario la economía decrecerá. Más todavía, un aumento en la media esperada de la productividad marginal del capital o en la intensidad del salto de dicha productividad, incrementan la tasa de crecimiento económico. Por su parte, la variabilidad en el componente determinista de la tasa de crecimiento es:

$$
E\left[\frac{d k_{t}}{k_{t}}\right]^{2}=\alpha^{2}\left(\sigma_{y}^{2}+\nu_{y}^{2} \theta_{y}\right) d t
$$

Lo anterior significa que la varianza en la tasa de crecimiento depende de la varianza en la productividad marginal del capital y de la varianza en el salto de la misma. Por el otro lado, el componente estocástico es:

$$
\Psi_{\mathrm{W}}=\sigma_{y} \xi
$$


La ecuación anterior muestra que cada incremento temporal en la economía afectará directamente a la desviación estándar estocástica de la tasa de crecimiento.

\subsection{Bienestar económico}

Para verificar cuál es el efecto que tienen las variables fundamentales de la economía sobre el bienestar económico es necesario recurrir a la ecuación (2), sólo que considerando que para cualquier momento $t$ dicha ecuación cumple con lo siguiente:

$$
\mathcal{V}_{t}=E\left\{\int_{t}^{\infty} \ln c_{t} e^{-\rho t} d s \mid \mathcal{F}_{t}\right\}=\mathcal{J}\left(k_{t}, t\right)=V\left(k_{t}\right) e^{-\rho t}=\left(\beta_{0}+\beta_{1} \ln k_{t}\right) e^{-\rho t},
$$

es decir, mide la utilidad indirecta de los agentes. Por lo tanto, la ecuación del bienestar económico de los hogares, exactamente en $t=0$, es el resultado de la sustitución de las condiciones de equilibrio (16) y (17) en (24), de tal forma que:

$$
\mathcal{W}=\mathcal{J}\left(k_{t}, 0\right)=\frac{1}{\rho}\left(\ln \rho+\frac{\alpha}{\rho}+\ln k_{0}+\frac{\alpha \theta_{y}}{\rho} \ln \left(1+\nu_{y}\right)-\frac{\alpha^{2} \sigma_{y}^{2}}{2 \rho}-1\right) .
$$

Ahora se realiza un análisis de estática comparativa:

$$
\begin{gathered}
\frac{\partial \mathcal{W}}{\partial \alpha}=\frac{1+\theta_{y} \ln \left(1+\nu_{y}\right)-\alpha \sigma_{y}^{2}}{\rho^{2}}>0, \\
\frac{\partial \mathcal{W}}{\partial \theta_{y}}=\frac{\alpha \ln \left(1+\nu_{y}\right)}{\rho^{2}}>0, \\
\frac{\partial \mathcal{W}}{\partial \sigma_{y}}=-\frac{\alpha^{2} \sigma_{y}}{\rho^{2}}<0 .
\end{gathered}
$$

De las ecuaciones (26)-(28) se deduce que incrementos en la media esperada de la productividad marginal del capital (siempre que $\theta_{y}>\sigma_{y}^{2}$ ), así como en la intensidad del salto en la productividad, tienen un impacto positivo en el bienestar económico de los hogares. Sin embargo, incrementos en la desviación estándar de la productividad marginal del capital genera efectos negativos sobre el bienestar económico.

\section{Sistema financiero}

Dentro de los múltiples objetivos que persigue el sistema financiero de una economía, uno de los más importantes es la canalización de recursos provenientes de unidades o agentes económicos que registran superávits, al término de sus actividades económicas de cada periodo, hacia otras unidades o agentes económicos que hayan registrado déficits. Basado en esta idea, el análisis realizado en este apartado introduce a la economía el sistema financiero.

\subsection{Aparato productivo}

Para introducir el sistema financiero, es necesario modificar un poco el comportamiento de la economía ya descrito en el aparato anterior, en 
particular respecto a las dotaciones iniciales de los agentes, así que suponga que el sector productivo de la economía ahora está compuesto por dos tipos de productores, a saber: un primer productor que posee el capital necesario para realizar sus actividades de producción, más un remanente resultado de sus superávits registrados en periodos previos, y un segundo productor que no posee el capital necesario para producir.

Lo anterior implica que del total de la población de la economía, $N$, que se supone permanece constante periodo tras periodo, los hogares dueños de los medios de producción son de dos tipos, los prestamistas (l) y los prestatarios (b), es decir, $N=N^{l}+N^{b}$. Los agentes prestamistas tienen un stock de capital mayor al de los agentes prestatarios y de manera conjunta componen el stock de capital total de la economía, de tal suerte que $K_{t}=K_{t}^{l}+K_{t}^{b}$ con $K_{t}^{l}>K_{t}^{b}$. Ambos agentes representativos, productor prestamista y prestatario, necesitan un nivel $k_{t}$ para llevar a cabo el proceso productivo (véase ecuación 8), el primero de ellos posee:

$$
k_{t}^{l}=k_{t}+\tilde{k}_{t}
$$

en donde $\tilde{k}_{t}$ mide el nivel de capital adicional con $\tilde{k}_{0}>0$, mientras que el segundo posee menos de lo necesario para producir. Bajo estas condiciones, el productor prestamista puede decidir otorgar bajo la forma de crédito su capital adicional, dando lugar al sistema financiero. Dado que es de interés analizar exclusivamente el efecto del sistema financiero sobre el crecimiento, por simplicidad en el análisis suponga que el sistema financiero tiene como único objetivo la reasignación del capital sin la obtención de ganancia alguna y no realiza ninguna otra actividad. Esto permite que el productor prestatario acuda a solicitar en préstamo el capital que le falta para realizar sus actividades de producción. Se supone que la cantidad de capital que el agente prestamista coloca en el sistema financiero es exactamente igual a la cantidad de capital requerida por el agente prestatario, de tal forma que no existen recursos ociosos en la economía. Esto implica que para producir el productor prestatario necesita:

$$
k_{t}=k_{t}^{b}+\tilde{k}_{t} .
$$

Se supondrá que existe un costo por el crédito, ${ }^{1}$ de tal forma que ahora el capital $\tilde{k}_{t}$ puede ser adquirido pagando un costo $\delta>0$, y cuya dinámica es:

$$
d R_{k}=\frac{d \tilde{k}_{t}}{\tilde{k}_{t}}=\delta\left(d t+\sigma_{\delta} d X_{t}+\nu_{\delta} d Q_{t}\right)
$$

en donde, $d R_{k}$ mide el rendimiento del capital disponible en el sistema financiero, $\delta$ es el rendimiento medio esperado, $\sigma_{\gamma}$ representa la volatilidad en el rendimiento, $\nu_{\delta}$ un salto en el rendimiento medio esperado, $d X_{t}$ es otro proceso de Wiener definido en un espacio fijo de probabilidad cuya filtración aumentada cumple que $\left(\Omega, F,\left(F_{t}\right)_{t \geq 0}, \mathbb{P}\right)$, con incrementos temporales independientes,

\footnotetext{
1 La introducción del costo de capital se hace de manera análoga a lo propuesto por Rivas-Aceves y Martínez (2009), modificando el comportamiento del costo.
} 
media cero y varianza igual al incremento temporal, mientras que $d Q_{t}$ es un nuevo proceso de Poisson que caracteriza la dinámica del salto en el rendimiento del capital, con una intensidad $\theta_{\delta}$ de manera que:

$\left\{\begin{array}{l}\operatorname{Pr}(\text { un salto durante } d t)=\operatorname{Pr}\left(d Q_{t}=1\right)=\theta_{\delta} d t \\ \operatorname{Pr}(\text { ningun salto durante } d t)=\operatorname{Pr}\left(d Q_{t}=0\right)=1-\theta_{\delta} d t+o(d t), \\ \operatorname{Pr}(\text { ms de un salto durante } d t)=\operatorname{Pr}\left(d Q_{t}>1\right)=o(d t)\end{array}\right.$

Los procesos $d X_{t}$ y $d Q_{t}$ no están correlacionados y el número inicial de saltos en el rendimiento del capital es $Q_{0}=0$. En consecuencia, el prestamista obtendrá al final del periodo $\tilde{k}_{t}+d R_{k} \tilde{k}_{t}=\tilde{k}_{t}\left(1+d R_{k}\right)$ y evidentemente, el prestatario pagará esa misma cantidad. Con ello, las restricciones presupuestales de cada agente se modifican de tal manera que para prestamista y prestatario, respectivamente, se tiene:

$$
\begin{gathered}
\frac{d k_{t}^{l}}{k_{t}^{l}}=\alpha\left(d t+\sigma_{y} d W_{t}+\nu_{y} d Z_{t}\right)+\tilde{k}_{t}\left(1+d R_{k}\right)-c_{t}^{l} d t \\
\frac{d k_{t}^{b}}{k_{t}^{b}}=\alpha\left(d t+\sigma_{y} d W_{t}+\nu_{y} d Z_{t}\right)-\tilde{k}_{t}\left(1+d R_{k}\right)-c_{t}^{b} d t .
\end{gathered}
$$

$\mathrm{Al}$ sustituir (31) en (35) y (36) se obtiene:

$$
\begin{gathered}
d k_{t}^{l}=k_{t}^{l}\left(\alpha+\delta-\frac{c_{t}^{l}}{k_{t}^{l}}\right) d t+k_{t}^{l}\left(\alpha \sigma_{y}+\delta \sigma_{\delta}\right) d U_{t}^{l}+k_{t}^{l}\left(\alpha \nu_{y}+\delta \nu_{\delta}\right) d M_{t}^{l}, \\
d k_{t}^{b}=k_{t}^{b}\left(\alpha-\delta-\frac{c_{t}^{b}}{k_{t}^{b}}\right) d t+k_{t}^{b}\left(\alpha \sigma_{y}-\delta \sigma_{\delta}\right) d U_{t}^{b}+k_{t}^{b}\left(\alpha \nu_{y}-\delta \nu_{\delta}\right) d M_{t}^{b},
\end{gathered}
$$

de donde $d U_{t}$ y $d M_{t}$ son los procesos de difusión y de Poisson resultantes de la modificación de la ecuación de acumulación del capital, respectivamente para prestamistas y prestatarios, y además todos los procesos involucrados no están correlacionados entre sí. Ahora, la toma de decisiones de los agentes representativos considera de manera simultánea las restricciones $\left(35^{\prime}\right)$ y $\left(36^{\prime}\right)$, así como la información disponible en $\mathrm{t}=0$ determinada por $\mathcal{F}_{0}=\left\{k_{0}, Z_{0}, \tilde{k}_{0}, Q_{0}, M_{0}\right\}$.

\subsection{Equilibrio macroeconómico}

Para determinar el equilibrio macroeconómico se necesita establecer el equilibrio del sector de los hogares, que bajo las condiciones establecidas es igual para prestamistas y prestatarios y se rige bajo el mismo esquema que el establecido en el apartado anterior, así como el equilibrio del aparato productivo que depende de los equilibrios individuales de ambos tipos de productores.

\subsubsection{Equilibrio del agente prestamista}

El agente prestamista toma sus decisiones con base en las condiciones establecidas por la ecuación (2) sujeta a la restricción (35'), cuya condición Hamilton-Jacobi-Bellman igualada a cero es la siguiente, (véase el apéndice matemático): 


$$
\begin{aligned}
0 & \left.=\ln c_{t}^{l}-\rho V\left(k_{t}^{l}\right)+k_{t}^{l} V^{\prime}\left(k_{t}^{l}\right)\left(\alpha+\delta-\frac{c_{t}^{l}}{k_{t}^{l}}\right)+\frac{1}{2} V^{\prime \prime}\left(k_{t}^{l}\right) k_{t}^{l^{2}} \alpha^{2} \sigma_{y}^{2}+\delta^{2} \sigma_{\delta}^{2}\right) \\
& +\left[V\left(k_{t}^{l}\left(1+\alpha \nu_{y}+\delta \nu_{\delta}\right)\right)-V\left(k_{t}^{l}\right)\right] d M_{t}^{l} .
\end{aligned}
$$

La ecuación anterior tiene las siguientes condiciones de optimalidad:

$$
\begin{gathered}
\frac{\partial H J B}{\partial c_{t}^{l}}=\frac{1}{c_{t}^{l}}-V^{\prime}\left(k_{t}^{l}\right)=0, \\
V\left(k_{t}^{l}\right)=\beta_{0}^{l}+\beta_{1}^{l} \ln k_{t}^{l}, \\
V^{\prime}\left(k_{t}^{l}\right)=\frac{\beta_{1}^{l}}{k_{t}^{l}}, \\
V^{\prime \prime}\left(k_{t}^{l}\right)=-\frac{\beta_{1}^{l}}{k_{t}^{l^{2}}}, \\
c_{t}^{l}=\frac{k_{t}^{l}}{\beta_{1}^{l}}, \\
\beta_{1}^{l}=\frac{1}{\rho} .
\end{gathered}
$$

Las ecuaciones (38) y (42) muestran que el agente prestamista consumirá siempre una proporción fija de su capital, por lo que un mayor nivel de capital resulta siempre en un mayor nivel de consumo. De las condiciones de optimalidad anteriores resulta:

$$
0=\ln \rho-\beta_{0}^{l} \rho+\frac{\alpha}{\rho}+\frac{\delta}{\rho}-\frac{\alpha^{2} \sigma_{y}^{2}+\delta^{2} \sigma_{\delta}^{2}}{2 \rho}-1+\frac{\alpha \theta_{y}}{\rho} \ln \left(1+\nu_{y}\right)+\frac{\delta \theta_{\delta}}{\rho} \ln \left(1+\nu_{\delta}\right) .
$$

Por lo tanto, el equilibrio del agente representativo de tipo prestamista es:

$$
\begin{gathered}
\beta_{1}^{l}=\frac{1}{\rho} \\
\beta_{0}^{l}=\frac{1}{\rho}\left[\ln \rho+\frac{\alpha+\delta}{\rho}+\frac{\alpha \theta_{y}}{\rho} \ln \left(1+\nu_{y}\right)+\frac{\delta \theta_{\delta}}{\rho} \ln \left(1+\nu_{\delta}\right)-\frac{\alpha^{2} \sigma_{y}^{2}+\delta^{2} \sigma_{\delta}^{2}}{2 \rho}-1\right] \\
k_{t}^{l}=k_{0}^{l} e^{\left(\alpha+\delta-\rho-\frac{\alpha^{2} \sigma_{y}^{2}+\delta^{2} \sigma_{\delta}^{2}}{2}\right) t+\left(\alpha \sigma_{y}+\delta \sigma_{y}\right) U_{t}^{l}+\ln \left(1+\alpha \nu_{y}\right) M_{t}+\ln \left(1+\delta \nu_{\delta}\right) M_{t}} \\
c_{t}^{l}=\rho k_{0}^{l} e^{\left(\alpha+\delta-\rho-\frac{\alpha^{2} \sigma_{y}^{2}+\delta^{2} \sigma_{\delta}^{2}}{2}\right) t+\left(\alpha \sigma_{y}+\delta \sigma_{y}\right) U_{t}^{l}+\ln \left(1+\alpha \nu_{y}\right) M_{t}+\ln \left(1+\delta \nu_{\delta}\right) M_{t}}
\end{gathered}
$$




$$
\begin{aligned}
\psi^{l} & =\left(\alpha+\delta-\rho-\frac{\alpha^{2} \sigma_{y}^{2}+\delta^{2} \sigma_{\delta}^{2}}{2}\right) t+\left(\alpha \sigma_{y}+\delta \sigma_{y}\right) \xi \\
& +\left[\ln \left(1+\alpha \nu_{y}\right)+\ln \left(1+\delta \nu_{\delta}\right)\right] M_{t}
\end{aligned}
$$

De este equilibrio se rescata lo siguiente: las proporciones $\beta_{1}^{l}$ y $\beta_{0}^{l}$ son invariantes en el tiempo, que el nivel de capital depende sólo del nivel de capital inicial y que el consumo per cápita está directamente determinado por las preferencias de los consumidores y por la trayectoria de equilibrio del capital.

\subsubsection{Equilibrio del agente prestatario}

El agente prestatario toma sus decisiones con base en las condiciones establecidas por la ecuación (2) sujeta a la restricción (36'), cuya condición Hamilton-Jacobi-Bellman de manera conjunta con sus condiciones de optimalidad, véase el apéndice matemático, son:

$$
\begin{gathered}
0=\ln c_{t}^{b}-\rho V\left(k_{t}^{b}\right)+k_{t}^{b} V^{\prime}\left(k_{t}^{b}\right)\left(\alpha-\delta-\frac{c_{t}^{b}}{k_{t}^{b}}\right)+\frac{1}{2} V^{\prime \prime}\left(k_{t}^{b}\right) k_{t}^{b^{2}}\left(\alpha^{2} \sigma_{y}^{2}-\delta^{2} \sigma_{\delta}^{2}\right) \\
+\left[V\left(k_{t}^{b}\left(1+\alpha \nu_{y}-\delta \nu_{\delta}\right)\right)-V\left(k_{t}^{b}\right)\right] d M_{t}^{b} \\
\frac{\partial H J B}{\partial c_{t}^{b}}=\frac{1}{c_{t}^{b}}-V^{\prime}\left(k_{t}^{b}\right)=0 \\
V\left(k_{t}^{b}\right)=\beta_{0}^{b}+\beta_{1}^{b} \ln k_{t}^{b} \\
V^{\prime}\left(k_{t}^{b}\right)=\frac{\beta_{1}^{b}}{k_{t}^{b}} \\
V^{\prime \prime}\left(k_{t}^{b}\right)=-\frac{\beta_{1}^{b}}{k_{t}^{b^{2}}} \\
c_{t}^{b}=\frac{k_{t}^{b}}{\beta_{1}^{b}} \\
\beta_{1}^{b}=\frac{1}{\rho}
\end{gathered}
$$

Análogamente que para el agentes prestamista, para el agente prestatario su nivel de consumo siempre será una proporción fija de su capital, tal y como lo muestran las ecuaciones (51) y (55). Asimismo, el nivel de consumo aumentará cuanto mayor sea su nivel de capital. De las condiciones de optimalidad anteriores se obtiene que:

$$
0=\ln \rho-\beta_{0}^{b} \rho+\frac{\alpha}{\rho}-\frac{\delta}{\rho}-\frac{\alpha^{2} \sigma_{y}^{2}-\delta^{2} \sigma_{\delta}^{2}}{2 \rho}-1+\frac{\alpha \theta_{y}}{\rho} \ln \left(1+\nu_{y}\right)-\frac{\delta \theta_{\delta}}{\rho} \ln \left(1+\nu_{\delta}\right)
$$


De esta manera el equilibrio para el agente prestatario es:

$$
\begin{gathered}
\beta_{1}^{b}=\frac{1}{\rho} \\
\beta_{0}^{b}=\frac{1}{\rho}\left[\ln \rho+\frac{\alpha}{\rho}+\frac{\alpha \theta_{y}}{\rho} \ln \left(1+\nu_{y}\right)-\frac{\delta}{\rho}-\frac{\alpha^{2} \sigma_{y}^{2}-\delta^{2} \sigma_{\delta}^{2}}{2 \rho}-\frac{\delta \theta_{\delta}}{\rho} \ln \left(1+\nu_{\delta}\right)-1\right] \\
k_{t}^{b}=k_{0}^{b} e^{\left(\alpha-\delta-\rho-\frac{\alpha^{2} \sigma_{y}^{2}-\delta^{2} \sigma_{\delta}^{2}}{2}\right) t+\left(\alpha \sigma_{y}-\delta \sigma_{y}\right) U_{t}^{l}+\ln \left(1+\alpha \nu_{y}\right) M_{t}-\ln \left(1+\delta \nu_{\delta}\right) M_{t}} \\
c_{t}^{b}=\rho k_{0}^{b} e^{\left(\alpha-\delta-\rho-\frac{\alpha^{2} \sigma_{y}^{2}-\delta^{2} \sigma_{\delta}^{2}}{2}\right) t+\left(\alpha \sigma_{y}-\delta \sigma_{y}\right) U_{t}^{l}+\ln \left(1+\alpha \nu_{y}\right) M_{t}-\ln \left(1+\delta \nu_{\delta}\right) M_{t}} \\
\psi^{b}=\left(\alpha-\delta-\rho-\frac{\alpha^{2} \sigma_{y}^{2}-\delta^{2} \sigma_{\delta}^{2}}{2}\right) t+\left(\alpha \sigma_{y}-\delta \sigma_{y}\right) \xi \\
+\left[\ln \left(1+\alpha \nu_{y}\right)-\ln \left(1+\delta \nu_{\delta}\right)\right] M_{t}
\end{gathered}
$$

Se puede verificar una vez más que las proporciones $\beta_{1}^{b}$ y $\beta_{0}^{b}$ son invariantes en el tiempo, que el nivel de capital del prestatario también depende sólo del nivel de capital inicial con que cuente y que su consumo per cápita está directamente determinado por sus preferencias y por la trayectoria de equilibrio del capital.

\subsection{Tasa de crecimiento económico}

La tasa de crecimiento de la economía, cuando existe mercado de capitales, depende del promedio del crecimiento de los sectores productivos prestamista y prestatario, de tal forma que al considerar las ecuaciones (35), (36), (49) y (62) el componente determinista del crecimiento es:

$$
\begin{aligned}
\Psi & =\frac{\psi^{l}+\psi^{b}}{2}=\frac{E\left[\frac{d k_{t}^{l}}{k_{t}^{l}} \cdot \frac{1}{d t}\right]+E\left[\frac{d k_{t}^{b}}{k_{t}^{b}} \cdot \frac{1}{d t}\right]}{2} \\
& =\frac{\left(\alpha+\delta+\alpha \nu_{y} \theta_{y}+\nu_{\delta} \theta_{\delta}-\rho\right)+\left(\alpha+\alpha \nu_{y} \theta_{y}-\nu_{\delta} \theta_{\delta}-\delta-\rho\right)}{2} .
\end{aligned}
$$

Equivalentemente,

$$
\Psi=\alpha+\alpha \nu_{y} \theta_{y}-\rho .
$$

La tasa de crecimiento de una economía sin sistema financiero es exactamente igual a una con sistema financiero, lo anterior se verifica porque las ecuaciones (21) y (64) son iguales. Esto significa que la economía crece al mismo ritmo cuando el sistema financiero permite la reasignación del capital de tal forma que todos los productores lleven a cabo sus procesos productivos. A primera 
vista puede parecer que el efecto del sistema financiero sobre el crecimiento es nulo, sin embargo esto no es así ya que depende del nivel que tome el costo del capital en el sistema financiero, escenario que se analizará en la siguiente sección.

Si el componente determinista en el crecimiento está dado por (64), entonces su variabilidad y la parte estocástica de la tasa de crecimiento son iguales a las condiciones establecidas en (22) y (23).

\subsection{Restricciones al crédito e ineficiencia del sistema financiero}

En el apartado anterior se mostró que la tasa de crecimiento económico de una economía con un sistema financiero es igual a la de una sin sistema financiero. Lo anterior se cumple siempre y cuando la única función del sistema financiero sea la de reasignar el capital no utilizado en la economía, sin embargo, si el sistema financiero amplía su campo de acción y busca realizar una diversidad de actividades con el objetivo de obtener alguna ganancia derivada del manejo de los recursos en él depositados, entonces se pueden generan ineficiencias al interior de dicho mercado, con lo que el costo del capital aumentaría. Esta misma consecuencia resulta de las restricciones al crédito, es decir, limitación en el uso del capital o poco capital disponible para el crédito productivo genera costos del capital elevados.

$\mathrm{Y}$ es precisamente un costo elevado lo que genera impactos negativos sobre el crecimiento. Considérese la restricción presupuestal del prestatario establecida por (35) y suponga que el costo del capital, determinado por la ecuación (31), es tan elevado que ocasione que dicha restricción sea igual a cero o negativa. En otras palabras que se cumpla que $\tilde{k}_{t}\left(1+d R_{k}\right)-c_{t}^{b} d t \geq$ $\alpha\left(d t+\sigma_{y} d W_{t}+\nu_{y} d Z_{t}\right)$, si esto es así, entonces existen dos posibles escenarios, a saber: el primero se da cuando el productor desea mantener fijo su nivel de consumo y, por tanto, un elevado costo en el capital lo lleva a sacrificar el pago de su deuda, y el segundo consiste en mantener el pago de la deuda a costa de sacrificar su consumo. Bajo cualquier escenario, el productor tiene incentivos para no llevar a cabo sus actividades económicas porque de lo contrario sería no óptimo hacerlo toda vez que no se obtendrían las ganancias necesarias para subsistir, lo que se traduce en una caída de la tasa de crecimiento ya que el sector prestatario no produciría. Si esto es así, la tasa de crecimiento de la economía sería:

$$
\Psi=\frac{\alpha+\alpha \nu_{y} \theta_{y}-\rho}{N^{l}} .
$$

Evidentemente, la tasa de crecimiento establecida en (65) es menor que la establecida en (64), es decir $\alpha+\alpha \nu_{y} \theta_{y}-\rho / N^{l}<\alpha+\alpha \nu_{y} \theta_{y}-\rho$. Es en este caso cuando el sistema financiero tiene su efecto negativo sobre el crecimiento. En consecuencia, un aumento desmedido en el costo de capital ocasionado por restricciones al crédito o por ineficiencias del sistema financiero, que genere desincentivos en el aparato productivo, genera una caída en la tasa de crecimiento, y además desempleo en los factores de la producción. El aumento puede medirse tanto por el costo medio esperado $\delta$, por el componente volátil $\sigma_{\delta}$, como por los saltos inesperados medidos por $\nu_{\delta}$. 


\subsection{Bienestar económico}

Con la existencia de dos tipos de hogares, prestamistas y prestatarios, el bienestar económico en toda la economía depende del bienestar económico de cada uno de ellos. Por lo tanto, al considerar para cada agente que:

$$
\begin{aligned}
& \mathcal{V}_{t}^{l}=E\left\{\int_{t}^{\infty} \ln c_{t}^{l} e^{-\rho t} d s \mid \mathcal{F}_{t}\right\}=\mathcal{J}\left(k_{t}^{l}, t\right)=V\left(k_{t}^{l}\right) e^{-\rho t}=\left(\beta_{0}^{l}+\beta_{1}^{l} \ln k_{t}^{l}\right) e^{-\rho t}, \\
& \mathcal{V}_{t}^{b}=E\left\{\int_{t}^{\infty} \ln c_{t}^{b} e^{-\rho t} d s \mid \mathcal{F}_{t}\right\}=\mathcal{J}\left(k_{t}^{b}, t\right)=V\left(k_{t}^{b}\right) e^{-\rho t}=\left(\beta_{0}^{b}+\beta_{1}^{b} \ln k_{t}^{b}\right) e^{-\rho t},
\end{aligned}
$$

como la utilidad indirecta de ambos agentes y si se sustituyen (45) y (46) en (66), así como (58) y (59) en (67), entonces la ecuación del bienestar económico en $t=0$ es:

$$
\begin{aligned}
\mathcal{W}= & \mathcal{J}\left(k_{t}, 0\right)=\frac{1}{\rho}\left[\ln \rho+\frac{\alpha+\delta}{\rho}+\frac{\alpha \theta_{y}}{\rho} \ln \left(1+\nu_{y}\right)+\frac{\delta \theta_{\delta}}{\rho} \ln \left(1+\nu_{\delta}\right)+k_{0}^{l}-\frac{\alpha^{2} \sigma_{y}^{2}+\delta^{2} \sigma_{\delta}^{2}}{2 \rho}-1\right] \\
& +\frac{1}{\rho}\left[\ln \rho+\frac{\alpha}{\rho}+\frac{\alpha \theta_{y}}{\rho} \ln \left(1+\nu_{y}\right)+k_{0}^{b}-\frac{\delta}{\rho}-\frac{\alpha^{2} \sigma_{y}^{2}-\delta^{2} \sigma_{\delta}^{2}}{2 \rho}-\frac{\delta \theta_{\delta}}{\rho} \ln \left(1+\nu_{\delta}\right)-1\right]
\end{aligned}
$$

El análisis de estática comparativa dice que:

$$
\begin{gathered}
\frac{\partial \mathcal{W}}{\partial \alpha}=\frac{2\left[1+\theta_{y} \ln \left(1+\nu_{y}\right)-\alpha \sigma_{y}^{2}\right]}{\rho^{2}}>0, \\
\frac{\partial \mathcal{W}}{\partial \sigma_{y}}=-\frac{2 \alpha^{2} \sigma_{y}}{\rho^{2}}<0 \\
\frac{\partial \mathcal{W}}{\partial \theta_{y}}=\frac{2 \alpha\left[\ln \left(1+\nu_{y}\right)\right]}{\rho^{2}}>0 \\
\frac{\partial \mathcal{W}}{\partial \delta}=\frac{\partial \mathcal{W}}{\partial \sigma_{\delta}}=\frac{\partial \mathcal{W}}{\partial \theta_{\delta}}=0
\end{gathered}
$$

De lo anterior se deduce que, el bienestar económico se ve afectado de manera positiva cuando se presentan incrementos en la productividad media esperada (siempre que se cumpla que $\theta_{y}>\alpha \sigma_{y}^{2}$ ), así como cuando aumenta la intensidad del salto en la productividad marginal del capital. Sin embargo el bienestar económico cae cada vez que la variabilidad en la productividad aumenta. Es importante señalar que modificaciones en el rendimiento del capital no impacta sobre el bienestar económico, siempre y cuando el sistema financiero tenga como única función reasignar el capital ocioso.

En caso contrario, el costo será tan elevado que el bienestar económico de la economía estará determinado sólo por el de los hogares prestamistas, el cual es claro es menor que el mostrado en (68). Por ende, es deseable que el costo 
del capital se mantenga al alcance de los productores prestatarios, desafortunadamente las ineficiencias en el sistema financiero tienden a incrementar dicho costo, por lo que una posible forma de evitarlo es mediante la intervención del gobierno, escenario que se analiza a continuación.

\section{Regulación gubernamental}

Ya se sabe que si el productor prestatario no obtiene el capital que le falta para llevar a cabo sus actividades económicas, entonces la tasa de crecimiento se verá afectada de manera negativa, y esto solo puede ocurrir si el costo del capital es tan alto que la acumulación del capital en el sector prestatario se vuelva nula o negativa. Para evitar dicha caída en la tasa de crecimiento, se debe cumplir que:

$$
d y_{t}^{b}-\tilde{k}_{t}\left(1+d R_{k}\right)-c_{t}^{b} d t>0 .
$$

Al despejar para el costo del capital se obtiene:

$$
d R_{k}<\frac{d y_{t}^{b}-c_{t}^{b} d t}{k_{t}^{b}}-1
$$

En el equilibrio, ecuaciones (3), (60) y (61), el costo del capital debe cumplir siempre que:

$$
d R_{k}<\alpha\left(d t+\sigma_{y} d W_{t}+\nu_{y} d Z_{t}\right)-\rho-1 .
$$

La ecuación anterior muestra que, si el costo del capital es menor que la productividad marginal media esperada del productor prestatario descontando la tasa subjetiva de descuento, entonces este agente decidirá contratar el crédito y así realizar su proceso productivo. Al sustituir la ecuación (31) en (75), resulta que los componentes determinista y estocástico de los procesos involucrados deben respectivamente cumplir:

$$
\begin{gathered}
\left(\delta+\delta \theta_{\delta}\right) d t<\left(\alpha+\alpha \theta_{y}\right) d t, \\
\delta \sigma_{\delta} d X_{t}<\alpha \sigma_{y} d W_{t} . \\
\delta^{2} \sigma_{\delta}^{2} d t<\alpha^{2} \sigma_{y}^{2} d t .
\end{gathered}
$$

Suponga ahora que el gobierno interviene en la economía, a través de la regulación en el sistema financiero, con el único objetivo de evitar que el sector prestatario salga de las actividades económicas y así disminuya la tasa de crecimiento. Para lograrlo puede aplicar, en todo momento $t$ un impuesto $\tau_{\delta}$, el cual representa la forma bajo la cual el gobierno regula al sistema financiero, no se trata de una figura impositiva con el fin de obtener recursos para financiar un gasto. Dicho impuesto representa una proporción del costo del capital cada vez que (75) no se cumpla, por lo que:

$$
\tau_{\delta}\left[\left(\delta+\delta \theta_{\delta}+\delta^{2} \sigma_{\delta}^{2}\right) d t\right] \geq\left(\alpha+\alpha \theta_{y}+\alpha^{2} \sigma_{y}^{2}\right) d t-\rho-1 .
$$

Asimismo, al definir un límite inferior $\omega$ se puede determinar que el impuesto al costo de capital, en presencia de un sistema financiero ineficiente, es: 


$$
\tau_{\delta}=\frac{\left(\alpha+\alpha \theta_{y}+\alpha^{2} \sigma_{y}^{2}\right) d t-\rho-1}{\left(\delta+\delta \theta_{\delta}+\delta^{2} \sigma_{\delta}^{2}\right) d t}+\omega .
$$

Adicionalmente se supone que el gobierno no realiza ninguna otra actividad, por lo que su restricción presupuestal está dada por:

$$
g d t=\tau_{\delta},
$$

en donde $g$ mide el nivel de gasto medio esperado per cápita que realiza el gobierno en forma de subsidio a la producción, exclusivamente para el sector prestatario. Con ello, ante un aumento desmedido en el costo del capital, el gobierno evita la caída en la tasa de crecimiento mediante incentivos a la producción del sector prestatario a través de subsidios financiados precisamente por el rendimiento del capital bajo ambientes ineficientes del sistema financiero, crisis financieras, restricciones al crédito, o cualquier otro escenario que eleve el costo del capital por encima de lo deseado.

\subsection{Comportamiento óptimo del impuesto al costo del capital}

Como ya se analizó, la intervención gubernamental aparece cuando el costo del capital es excesivamente alto mediante la figura de un impuesto al rendimiento del capital. Ahora bien, el impuesto lo debe pagar alguien y como sólo el sistema financiero o el agente prestamista son sujetos de tal fenómeno, entonces surgen dos escenarios posibles. El primero sucede cuando el sistema financiero busca la obtención de ganancias, debido al arbitrio del capital en su poder, lo que ocasiona dos tipos de rendimientos del capital: el que el sistema financiero obtiene $y$ el que el prestamista recibe del sistema financiero, evidentemente con una brecha existente entre ambos siendo menor el rendimiento que el prestamista recibe. Si esto es así, entonces el sistema financiero debe asumir el costo del impuesto.

El segundo escenario tiene origen cuando es precisamente el prestamista el que exige un alto pago por el préstamo de su capital remanente, bajo este escenario el impuesto debe ser asumido por él. Sin embargo, dicho impuesto no puede alcanzar niveles altos tal que ocasione que la acumulación del capital del sector prestamista sean nula o negativa, ya que de ser así la tasa de crecimiento de dicho sector se vería afectada de manera negativa. En consecuencia, se debe cumplir que:

$$
d y_{t}^{l}+\tilde{k}_{t}\left(1+d R_{k}\right)-\tau_{\delta} \tilde{k}_{t} d R_{k}-c_{t}^{l} d t>0 .
$$

$\mathrm{Si}$ a la ecuación anterior se le sustituyen las condiciones de equilibrio (3), (47), (48), se consideran los componentes determinista y estocástico de los procesos que representan tanto al rendimiento del capital como al del producto, y posteriormente se despeja para el impuesto al rendimiento del capital, entonces se obtiene:

$$
\tau_{\delta}<\frac{\left(\alpha+\delta+\alpha \theta_{y}+\delta \theta_{\delta}+\alpha^{2} \sigma_{y}^{2}+\delta^{2} \sigma_{\delta}^{2}\right) d t+1-\rho}{\left(\delta+\delta \theta_{\delta}+\delta^{2} \sigma_{\delta}^{2}\right) d t} .
$$

Dado que en la ecuación (79) el parámetro $\omega$ es desconocido, entonces el nivel de impuesto no se puede determinar ya dicho límite inferior depende del 
rendimiento del capital. Sin embargo, al considerar condiciones establecidas en (78) y (82) se puede determinar un intervalo óptimo, por unidad de tiempo, dentro del cual el impuesto al capital puede situarse, a saber:

$$
\frac{\alpha+\alpha \theta_{y}+\alpha^{2} \sigma_{y}^{2}-\rho-1}{\delta+\delta \theta_{\delta}+\delta^{2} \sigma_{\delta}^{2}} \leq \tau_{\delta}<\frac{\alpha+\delta+\alpha \theta_{y}+\delta \theta_{\delta}+\alpha^{2} \sigma_{y}^{2}+\delta^{2} \sigma_{\delta}^{2}+1-\rho}{\delta+\delta \theta_{\delta}+\delta^{2} \sigma_{\delta}^{2}}
$$

Cuando la intervención gubernamental sea necesaria a través de la regulación al sistema financiero, el impuesto al rendimiento del capital debe seguir el comportamiento óptimo determinado por la ecuación anterior, de esta manera se asegura la participación de los sectores prestamista y prestatario en las actividades económicas y, por ende, se evitara la caída en la tasa de crecimiento.

\subsection{Tasa de crecimiento con regulación financiera}

Hasta ahora se ha mostrado cómo el gobierno interviene en la economía si el sistema financiero presenta ineficiencias. Con dicha intervención las ecuaciones de acumulación del capital de los productores prestamistas y prestatarios se modifican, de tal forma que ahora se tiene:

$$
\begin{array}{r}
\frac{d k_{t}^{l}}{k_{t}^{l}}=\alpha\left(d t+\sigma_{y} d W_{t}+\nu_{y} d Z_{t}\right)+\tilde{k}_{t}\left(1+d R_{k}\right)-\tau_{\delta} \tilde{k}_{t} d R_{k}-c_{t}^{l} d t, \\
\frac{d k_{t}^{b}}{k_{t}^{b}}=\alpha\left(d t+\sigma_{y} d W_{t}+\nu_{y} d Z_{t}\right)+g d t-\tilde{k}_{t}\left(1+d R_{k}\right)-c_{t}^{b} d t .
\end{array}
$$

De esta manera, la tasa de crecimiento económico de tipo determinista es:

$$
\Psi=\frac{E\left[\frac{d k_{t}^{l}}{k_{t}^{t}} \cdot \frac{1}{d t}\right]+E\left[\frac{d k_{t}^{b}}{k_{t}^{b}} \cdot \frac{1}{d t}\right]}{2}=\alpha+\alpha \nu_{y} \theta_{y}-\rho-\frac{\tau_{\delta}\left(\delta+\nu_{\delta} \theta_{\delta}\right)-g}{2},
$$

en donde, de acuerdo a la restricción (80), los componentes públicos gasto de gobierno medio esperado y el impuesto al rendimiento del capital se anulan. Lo anterior implica que, la regulación gubernamental lo único que genera es que la economía se desenvuelva al ritmo de crecimiento establecido en el apartado 2.3. Más todavía, se puede verificar de manera clara que la intervención del gobierno sólo corrige las distorsiones generadas por la ineficiencia del sistema financiero sobre la economía. Por último, la variabilidad en la tasa de crecimiento sería:

$$
E\left[\frac{d k_{t}}{k_{t}}\right]^{2}=\left[\alpha^{2}\left(\sigma_{y}^{2}+\nu_{y}^{2} \theta_{y}\right)-\tau_{\delta}^{2} \delta^{2}\left(\sigma_{\delta}^{2}+\nu_{\delta}^{2} \theta_{\delta}\right)+g^{2}\right] d t
$$

Análogamente, la condición (80) anula los efectos de la regulación.

\subsection{Bienestar económico}

Bajo estas condiciones, el bienestar económico de los hogares permanece intacto e igual a la condición establecida en (68) toda vez que, como ya se mencionó, 
la intervención gubernamental sólo corrige las distorsiones financieras y, en consecuencia, los efectos sobre el bienestar establecidos por las ecuaciones (69)(72) se mantienen.

\section{Trayectorias de crecimiento con o sin regulación}

Hasta ahora, lo que se ha mostrado es que la economía se puede desenvolver bajo una senda de crecimiento balanceado con pleno empleo y de equilibrio, ya sea con presencia de un sistema financiero eficiente o bien con uno ineficiente pero regulado por el gobierno. Una posible trayectoria de crecimiento de una economía con características como las analizadas en los apartados anteriores, se puede establecer mediante una simulación de tipo Monte Carlo.

En este apartado se presentan simulaciones de dicho estilo realizadas a partir de la metodología Box-Muller, para 25 posibles trayectorias bajo escenarios de eficiencia financiera o regulación. Las simulaciones consideran las dos posibles relaciones existentes entre la productividad marginal del capital y el rendimiento financiero del mismo, las cuales fueron establecidas en los apartados 2 y 3 , y están resumidas por las condiciones (76) y (77). Asimismo tienen como condiciones iniciales tasas de crecimiento de entre $3.5 \%$ y $4 \%$, de acuerdo con el crecimiento promedio anual para los países del G7 y México establecido por el Banco Mundial (2011), presentan una duración de 360 periodos y los resultados se presentan a continuación:

Gráfica 1. Posibles trayectorias de crecimiento bajo regulación financiera

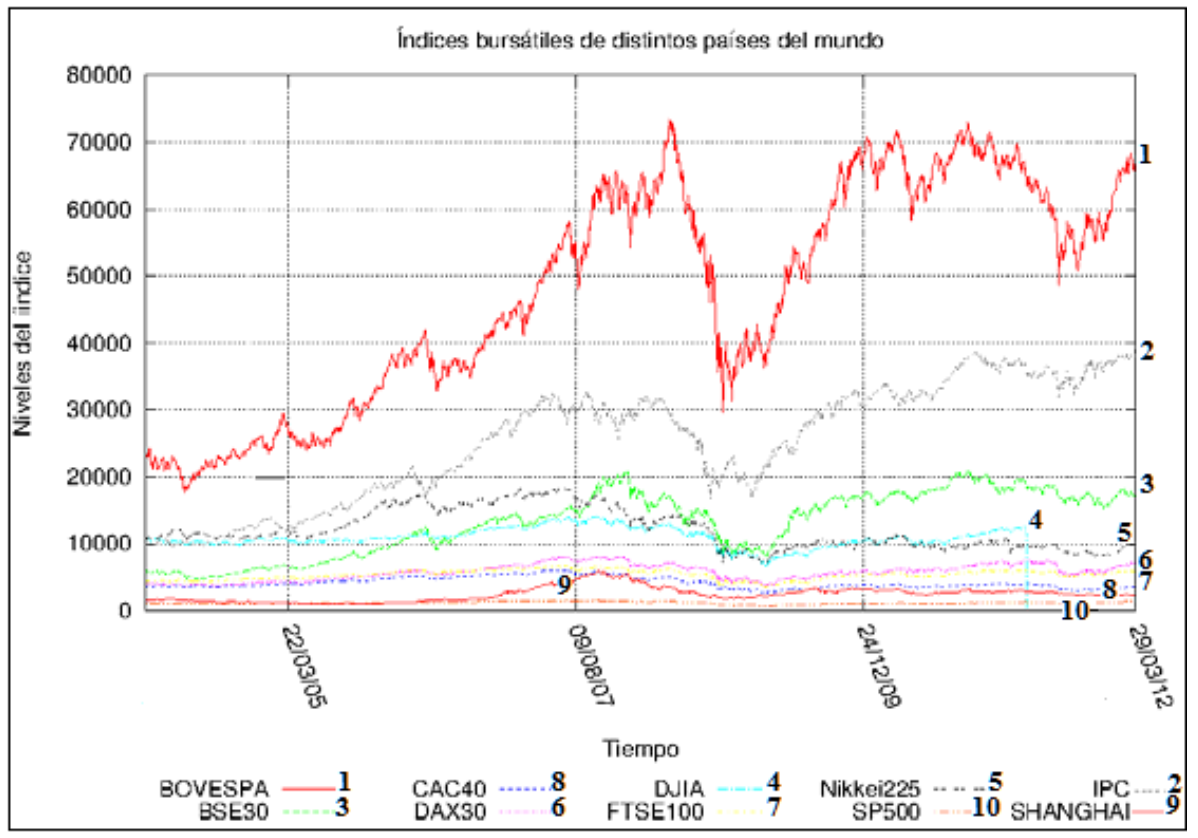

Fuente:Elaboración propia. 
Gráfica 2. Posibles trayectorias de crecimiento bajo ineficiencia financiera

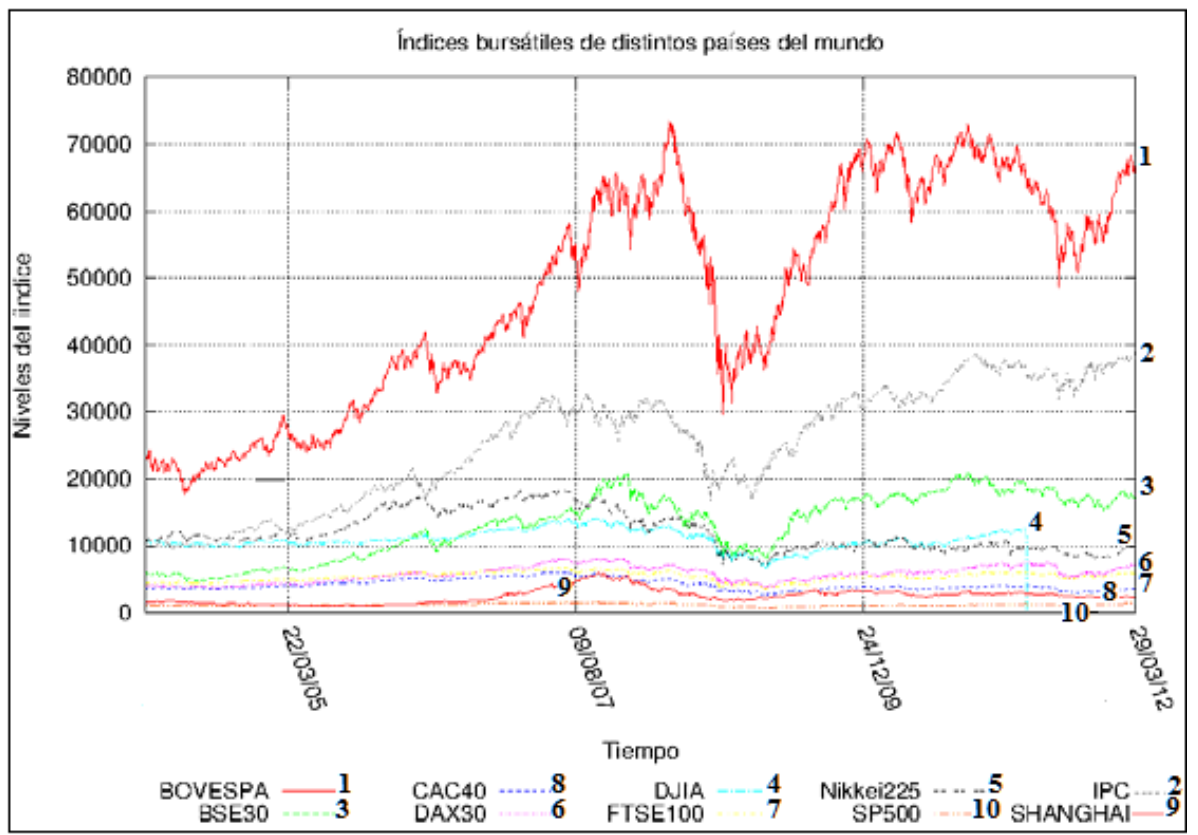

Fuente:Elaboración propia.

Como se puede apreciar en la Gráfica 1, el efecto de la regulación financiera sobre la tasa de crecimiento económico es positivo ya que se mantiene la condición que obliga al rendimiento financiero del capital estar por debajo de su productividad marginal, lo que concuerda con los resultados teóricos encontrados en esta investigación. En contraste, cuando el rendimiento financiero del capital es superior a su productividad marginal, entonces las posibles trayectorias del crecimiento tienden a ser menores en magnitud como lo muestra la Gráfica 2. Por lo tanto, la ineficiencia financiera afecta de manera negativa al crecimiento económico.

\section{Conclusiones}

A través de un modelo de crecimiento endógeno, de economía cerrada, con agentes económicos idénticos y bajo ambientes estocásticos, se mostró que el crecimiento económico depende de manera directa de la productividad marginal media esperada del capital, de la dispersión en la misma y de los saltos productivos, mientras que de manera negativa de la tasa subjetiva de descuento.

Al introducir inequidad en el nivel de ingreso, generada por superávits y déficits en los agentes participantes en las actividades productivas, la reasignación del capital remanente y desempleado resultante queda en manos del sistema financiero. Si el sistema financiero es eficiente, entonces la reasignación se lleva a cabo sin ningún problema y los sectores productivos prestamista y prestatario participan ambos en las actividades económicas, manteniendo así la tasa de crecimiento económico intacta. Por el contrario, si el sistema financiero 
presenta ineficiencias, o existen restricciones al crédito, etc., entonces el costo del capital se eleva de tal manera que deja fuera de las actividades productivas al sector prestatario, causando desempleo en los factores de la producción y, por tanto, afectando de manera negativa a la tasa de crecimiento económico.

Bajo este escenario, la intervención gubernamental vía la regulación financiera corrige las distorsiones creadas por dichas ineficiencias en el sistema financiero, garantiza la participación de ambos sectores en los procesos de producción y, por ende, mantiene la tasa de crecimiento de la economía en los niveles de equilibrio con pleno empleo. En particular, la intervención del gobierno se da por medio de un impuesto al rendimiento del capital cuando éste toma niveles excesivos. Los recursos captados por dicho impuesto son regresados al sistema bajo la forma de subsidios a la producción con el objetivo de fomentar que el sector productivo prestatario se mantenga en las actividades productivas.

Asimismo, se determina un comportamiento óptimo del impuesto sobre el rendimiento del capital que permita mantener el ritmo de crecimiento, gracias a que garantiza la participación de ambos sectores productivos en las actividades económicas. Es importante señalar que, la regulación gubernamental sólo corrige los efectos negativos del sistema financiero sobre la economía real y no genera ninguna otra distorsión sobre la economía. En otras palabras, la economía regresa a una senda de crecimiento sostenido, balanceado y de pleno empleo, gracias a la reasignación del capital.

Los resultados teóricos concuerdan con los resultados encontrados a partir de las simulaciones realizadas, bajo escenarios de ineficiencia financiera y regulación financiera, presentados en el apartado 4. Las simulaciones de tipo Monte Carlo muestran que las posibles trayectorias de crecimiento económico tienen sendas mayores en magnitud cuando el sistema financiero es eficiente o cuando hay regulación financiera, de tal manera que siempre se cumpla que la productividad marginal del capital sea mayor que su rendimiento financiero. En caso contrario, las simulaciones muestran que las posibles trayectorias de crecimiento económico serán menores en magnitud.

El análisis aquí realizado permite verificar que existe un vínculo entre la economía real y el sistema financiero, y que el buen funcionamiento de este último fomenta el crecimiento. Respecto del análisis, es importante decir que los resultados dependen de los supuestos establecidos por la cual es importante ampliarlo. En este sentido, la agenda pendiente señala que se debe modelar el efecto del sistema financiero internacional (economía abierta) sobre el crecimiento, ampliar el papel del gobierno en las actividades económicas así como introducir los impuesto al consumo y al ingreso para verificar los efectos del mismo en el crecimiento, y, finalmente, introducir el mercado de futuros, de opciones, etc., al interior del sistema financiero doméstico. 


\section{Apéndice Matemático}

\section{Derivación de la ecuación (9)}

El problema de control óptimo planteado por (2) y (8'), arroja que:

$$
\mathcal{J}\left(k_{t}, t\right)=\operatorname{Max}_{\left.c_{s}\right|_{[t, t+d t]}} E\left\{\ln \left(c_{t}\right) e^{-\rho t}+o(d t)+\mathcal{J}\left(k_{t}, t\right)+d \mathcal{J}\left(k_{t}, t\right) \mid \mathcal{F}_{t}\right\}
$$

Por su parte, el Lema de Îto establece que:

$$
\begin{aligned}
d \mathcal{J}\left(k_{t}, t\right) & =\left[\frac{\partial \mathcal{J}}{\partial t}+\frac{\partial \mathcal{J}}{\partial k_{t}} k_{t}\left(\alpha-\frac{c_{t}}{k_{t}}\right)+\frac{1}{2} \frac{\partial^{2} \mathcal{J}}{\partial k_{t}{ }^{2}} k_{t}{ }^{2} \sigma_{y}^{2}\right] d t+\frac{\partial \mathcal{J}}{\partial k_{t}} k_{t} \sigma_{y} d W_{t} \\
& +\frac{\partial \mathcal{J}}{\partial k_{t}} k_{t} v_{y} d Z_{t}
\end{aligned}
$$

Para encontrar la ecuación (9) se necesita sustituir $J\left(k_{t}, t\right)=V\left(k_{t}\right) e^{(\rho t)}$ y aplicar (A.2) a la ecuación (A.1), posteriormente tomar esperanzas y fijar $E(d z)=0$, dividir entre $d t$ y tomar el límite cuando $t \rightarrow 0$, con lo que se obtiene lo siguiente:

$$
\begin{aligned}
0 & =\ln c_{t}-\rho V\left(k_{t}\right)+k_{t} V^{\prime}\left(k_{t}\right)\left(\alpha-\frac{c_{t}}{k_{t}}\right)+\frac{1}{2} V^{\prime \prime}\left(k_{t}\right) k_{t}^{2} \sigma_{y}^{2} \\
& +\left[V\left(k_{t}\left(1+\nu_{y}\right)\right)-V\left(k_{t}\right)\right] d Z t .
\end{aligned}
$$

\section{Derivación de la ecuación (34)}

El problema de control óptimo planteado por (2) y (32'), arroja que:

$$
\mathcal{J}\left(k_{t}^{l}, t\right)=\operatorname{Max}_{\left.c_{s}\right|_{[t, t+d t]}} E\left\{\ln \left(c_{t}^{l}\right) e^{-\rho t}+o(d t)+\mathcal{J}\left(k_{t}^{l}, t\right)+d \mathcal{J}\left(k_{t}^{l}, t\right) \mid \mathcal{F}_{t}\right\}
$$

Por su parte, el Lema de Îto establece que:

$$
\begin{aligned}
d \mathcal{J}\left(k_{t}^{l}, t\right)= & {\left[\frac{\partial \mathcal{J}}{\partial t}+\frac{\partial \mathcal{J}}{\partial k_{t}^{l}} k_{t}^{l}\left(\alpha+\delta-\frac{c_{t}^{l}}{k_{t}^{l}}\right)+\frac{1}{2} \frac{\partial^{2} \mathcal{J}}{\partial k_{t}^{l^{2}}} k_{t}^{l^{2}}\left(\sigma_{y}^{2}+\sigma_{\delta}^{2}\right)\right] d t } \\
& +\frac{\partial \mathcal{J}}{\partial k_{t}^{l}} k_{t}^{l}\left(\sigma_{y}+\sigma_{\delta}\right) d U_{t}^{l}+\frac{\partial \mathcal{J}}{\partial k_{t}^{l}} k_{t}^{l}\left(v_{y}+\nu_{\delta}\right) d M_{t}^{l}
\end{aligned}
$$

Para encontrar la ecuación (34) se necesita sustituir $\mathcal{J}\left(k_{t}^{l}, t\right)=V\left(k_{t}^{l}\right) e^{-\rho t}$ y aplicar (A.5) a la ecuación (A.4), posteriormente tomar esperanzas y fijar $E\left(d M_{t}\right)=0$, dividir entre $d_{t}$ y tomar el límite cuando $t \rightarrow 0$, con lo que se obtiene lo siguiente:

$$
0=\ln c_{t}^{l}-\rho V\left(k_{t}^{l}\right)+k_{t}^{l} V^{\prime}\left(k_{t}^{l}\right)\left(\alpha+\delta-\frac{c_{t}^{l}}{k_{t}^{l}}\right)+\frac{1}{2} V^{\prime \prime}\left(k_{t}^{l}\right) k_{t}^{l^{2}}\left(\sigma_{y}^{2}+\sigma_{\delta}^{2}\right)
$$




$$
+\left[V\left(k_{t}^{l}\left(1+\nu_{y}+\nu_{\delta}\right)\right)-V\left(k_{t}^{l}\right)\right] d M_{t}^{l} .
$$

\section{Derivación de la ecuación (54)}

El problema de control óptimo planteado por (2) y (33'), arroja que:

$$
\mathcal{J}\left(k_{t}^{b}, t\right)=\operatorname{Max}_{\left.c_{s}\right|_{[t, t+d t]}} E\left\{\ln \left(c_{t}^{b}\right) e^{-\rho t}+o(d t)+\mathcal{J}\left(k_{t}^{b}, t\right)+d \mathcal{J}\left(k_{t}^{b}, t\right) \mid \mathcal{F}_{t}\right\}
$$

Por su parte, el Lema de Îto establece que:

$$
\begin{aligned}
d \mathcal{J}\left(k_{t}^{b}, t\right) & =\left[\frac{\partial \mathcal{J}}{\partial t}+\frac{\partial \mathcal{J}}{\partial k_{t}^{b}} k_{t}^{b}\left(\alpha-\delta-\frac{c_{t}^{b}}{k_{t}^{b}}\right)+\frac{1}{2} \frac{\partial^{2} \mathcal{J}}{\partial k_{t}^{b^{2}}} k_{t}^{b^{2}}\left(\sigma_{y}^{2}-\sigma_{\delta}^{2}\right)\right] d t \\
& +\frac{\partial \mathcal{J}}{\partial k_{t}^{b}} k_{t}^{b}\left(\sigma_{y}-\sigma_{\delta}\right) d U_{t}^{l}+\frac{\partial \mathcal{J}}{\partial k_{t}^{b}} k_{t}^{b}\left(v_{y}-\nu_{\delta}\right) d M_{t}^{b}
\end{aligned}
$$

Para encontrar la ecuación (54) se necesita sustituir $\mathcal{J}\left(k_{t}^{b}, t\right)=V\left(k_{t}^{b}\right) e^{-\rho t}$ y aplicar (A.8) a la ecuación (A.7), posteriormente tomar esperanzas y fijar $E\left(d M_{t}\right)=0$, dividir entre dt y tomar el límite cuando $t \rightarrow 0$, con lo que se obtiene lo siguiente:

$$
\begin{aligned}
0 & =\ln c_{t}^{b}-\rho V\left(k_{t}^{b}\right)+k_{t}^{b} V^{\prime}\left(k_{t}^{b}\right)\left(\alpha-\delta-\frac{c_{t}^{l}}{k_{t}^{b}}\right)+\frac{1}{2} V^{\prime \prime}\left(k_{t}^{b}\right) k_{t}^{b^{2}}\left(\sigma_{y}^{2}-\sigma_{\delta}^{2}\right) \\
& +\left[V\left(k_{t}^{b}\left(1+\nu_{y}-\nu_{\delta}\right)\right)-V\left(k_{t}^{b}\right)\right] d M_{t}^{b} .
\end{aligned}
$$

\section{Bibliografía}

Aghion, P. and P. Bolton, (1997). A Trickle Down Theory of Growth and Development, Review of Economic Studies, 64, pp. 151-172.

Bagehot, W. (1873). Lombard Street, a Description of the Money Market, London: Kegan Paul. Rpt., London: John Murray, 1920.

Banco Mundial, (2011). Indicadores de Política Económica y Deuda, http://datos.bancomundial.org/indicador/NY.GDP.MKTP.KD.ZG, recuperados el 19 de octubre 2011.

Banerjee, A. and A. Newman, (1993). Occupational Choice and the Process of Development, Journal of Political Economy, 101, pp. 274-298.

Barth, J.R., Caprio, G. Jr. y R. Levine, (2001a). The Regulation and Supervision of Banks around the World: A New Database, In Litan, R.E. y R. Herring eds., BrookingWharton Papers on Financial Services. Brookings Institution, Washington, DC, pp. $83-250$.

Barth, J.R., Caprio, G. Jr. y R. Levine, (2001b). Banking System around the Globe: Do Regulation and Ownership Affect Performance and Stability, In Mishkin, F.S. Ed., Prudential Supervision: What Works and What Dosen't, University of Chicago Press, Chicago, IL, pp.31-96.

Barth, J.R., Caprio, G. Jr. y R. Levine, (2004). Bank Regulation and Supervision: What Works Best?. Journal of Financial Intermediation, 13, pp. 205-248.

Barth, J.R., Caprio, G. Jr. y R. Levine, (2005). Rethinking Bank Supervision and Regulation: Until Angels Govern, Cambridge University Press, Cambridge, UK. 
Benabou, R. (1993). Workings of a City: Location, Education and Production, Quarterly Journal of Economics, 108, pp. 619-652.

Benabou, R, (1996a). Equity and Efficiency in Human Capital Investment: The Local Connection. Review of Economic Studies, 62(2), pp. 237-264.

Benabou, R. (1996b). Inequality and Growth, NBER Working Paper 5658, 1-56.

Bencivenga, V. y B. Smith, (1992). Deficits, Inflation and the Banking System in Developing Countries: The Optimal Degree of Financial Repression, Oxford Economics Papers 44, pp. $767-790$.

Durlauf, S. (1996a). A Theory of Persistent Income Inequality, Journal of Economic Growth, 1(1), pp. $75-94$.

Durlauf, S. (1996b). Neighborhood Feedbacks, Endogenous Stratification, and Income Inequality, forthcoming in "Proceedings of the Sixth International Symposium on Economic Theory and Econometrics, W. Barnett, G. Gandolfo y C. Hillinger, eds., Cambridge University Press.

Fernández, R. y R. Rogerson, (1994). Public Education and the Dynamics of Income Distribution: A Quantitative Evaluation of Education Finance Reform, NBER Working Paper 4883.

Fernández, R. y R. Rogerson, (1996). Income Distribution, Communities and the Quality of Public Education, Quarterly Journal of Economics, 111, pp. 135-164.

Galor, O. y J. Zeira, (1993). Income Distribution and Macroeconomics, Review of Economic Studies, 60, pp. 35-52.

Gurley, J. G., y E. S. Shaw, (1995). Financial Aspects of Economic Development, American Economic Review, 45, pp. 515-538.

Huybens, E. y R. Smith, (1999). Inflation, Financial Markets and Long-run Real Activity. Journal of Monetary Economics, 43, pp. 283-315.

Kremer M. y E. Maskin (1994). Segregation of Workers by Skill and the Rise of Inequality, Working Paper Massachusetts Institute of Technology.

Kunieda, T. (2008). Macroeconomics for Credit Market Imperfections and Heterogeneous Agents, Brown University Press.

La Porta, R., López-de-Silanes, F. y A. Shleifer, (2005), What Works in Security Laws?, Journal of Finance,

Mckinnon, R. I. (1973). Money and Capital in Economic Development, Washington, DC: Brookings Institution.

Piketty, T. (1997). The Dynamics of Wealth Distribution and the Interest Rate with Credit Rationing, Review of Economic Studies, 64, pp. 173-189.

Rivas-Aceves S. y J. F. Martínez Pérez, (2009). El Impacto de la Regulación Gubernamental en el Mercado de Capitales sobre el Crecimiento, capítulo en libro Economía teórica y aplicada, México y el mundo, Editorial UAM-Eón, México 2009, pp.59-68. ISBN UAM: 978-607-477-176-3, ISMB Eón: 978-607-7519-53-9.

Rivas-Aceves S. y J. F. Martínez Pérez, (2011). La Crisis Financiera Internacional y el Crecimiento Económico: La Banca de Desarrollo, una Posible Solución, capítulo en libro La integración económica: balance de un proceso inconcluso, Editorial Eón, México, 2011, pp. 73-98.

Roubini, N. y X. Sala-i-Martin, (1992). Financial Repression and Economic Growth. Journal of Development Economics, 39, pp. 5-30.

Roubini, N. y X. Sala-i-Martin, (1995). A Growth Model of Inflation, Tax Evasion and

Financial Repression. Journal of Monetary Economics, 35, pp. 275-310. 